\section{Abscopal Effect Following Radiation Therapy in Cancer Patients: A New Look from the Immunological Point of View}

\author{
Welsh J.1ْ, Bevelacqua J. J.2 ${ }^{2}$, Dobrzyński L. ${ }^{3}$, Mortazavi S. \\ A. R. ${ }^{4}$, Farjadian Sh. ${ }^{5}$, Mortazavi S. M. J..$^{6,7 *(1)}$
}

\begin{abstract}
Abscopal effect, a radiobiology term meaning "away from target", was practically unheard of just ten years ago. This effect describes the elimination and cure of a non-treated tumor when another part of the body is irradiated. Successful treatment of cancer in patients with multiple metastatic foci has sporadically been reported. Abscopal effect after radiotherapy has been introduced as the key factor which induced an anticancer response in these metastatic lesions. Moreover, not receiving chemotherapy is reported to have a role in cancer regression after radiotherapy. Given this consideration, it can be hypothesized that standard radiotherapy doses, which usually classified as high-dose, may cause cancer cells to expose or release their sequestered antigens that had been previously masked. Furthermore, radiotherapy can decrease the suppressive effect of regulatory $\mathrm{T}$ cells which usually down modulate immune responses against cancers. Moreover, some data show that low dose total-body irradiation (TBI) alone (without standard localized high dose radiotherapy) may cause suppression of distant metastasis of tumor cells. Induction of a "whole body abscopal effect" can be involved in suppression of distant metastasis. Here we discuss whether cancer treatments could be more successful if immune system is boosted, not destroyed by the treatments such as chemotherapy.
\end{abstract}

Citation: Welsh J, Bevelacqua JJ, Dobrzyński L, Mortazavi SAR, Farjadian Sh, Mortazavi SMJ. Abscopal Effect Following Radiation Therapy in Cancer Patients: A New Look from the Immunological Point of View.J Biomed Phys Eng. 2020;10(4):537-542. doi: 10.31661/jbpe.v0i0.1066.

Keywords

Immune System; Cancer; Radiotherapy; Radiobiology

\section{Introduction}

he abscopal effect was practically unheard of just ten years ago. Today, you cannot go to a radiation oncology conference and avoid hearing an overdose of talks and seeing a plethora of posters on the topic. There are lots of clinical trials underway or under review dealing with the abscopal effect and the integration of modern immunotherapy using high-dose ionizing radiation to induce this phenomenon. This is great both scientifically and medically and even promising for patients suffering from cancer. Abscopal comes from "ab scopus" means away from target, and is a radiobiology term that describes the elimination and cure of a non-treated tumor at a different location when irradiating a tumor in another part of the
${ }^{1} \mathrm{MD}, \mathrm{PhD}$, Department of Radiation Oncology,

Edward Hines Jr VA Hos-

pital, Hines, IL 60141,

United States

${ }^{2} \mathrm{PhD}$, Bevelacqua

Resources, Richland,

Washington 99352,

United States

${ }^{3} \mathrm{PhD}$, National Centre

for Nuclear Research,

Otwock-Świerk, Poland

${ }^{4} \mathrm{MD}$, School of Medi-

cine, Shiraz University

of Medical Sciences,

Shiraz, Iran

${ }^{5} \mathrm{PhD}$, Department of Immunology, School of

Medicine, Shiraz Univer-

sity of Medical Sciences,

Shiraz, Iran

${ }^{6} \mathrm{PhD}$, Department of

Medical Physics, School

of Medicine, Shiraz

University of Medical Sci-

ences, Shiraz, Iran

${ }^{7} \mathrm{PhD}$, Diagnostic Imag-

ing, Fox Chase Cancer

Center, Philadelphia,

Pennsylvania 19111,

United States

*Corresponding author:

S. M. J. Mortazavi

Department of Medical

Physics, School of Medi-

cine, Shiraz University

of Medical Sciences,

Shiraz, Iran

E-mail: mortazavismj@ gmail.com

Received: 5 December 2018 Accepted: 7 January 2019 
body [1-4]. In a report recently published by Azami et al., successful treatment of a 64-year old woman diagnosed with breast cancer with multiple bone, lung and lymph node metastases has been mentioned [5] She received local radiotherapy delivered to the breast mass and some of the bone metastases. However, this patient did not receive chemotherapy due to her poor performance status. The authors observed spontaneous cancer regression 10 months after radiotherapy, not only within the irradiated fields, but also in the non-exposed areas. They reported that all signs of cancer disappeared throughout the body and they stated that abscopal effect after radiotherapy was the key factor for the induction of anti-cancer response in all metastatic lesions. In 2014, Welsh also reported eradication of cancer in an athletic young man diagnosed with a progressive skin cancer [6]. Welsh believed that cancer can adversely affect the immune system, so that it cannot differentiate malignant cells from normal tissues and kill them properly. Moreover, recruitment of myeloid-derived suppressor cells (MDSC) and regulator $\mathrm{T}$ cells (Treg) by tumor strom prevent the activation of cytotoxic $\mathrm{T}$ cells and NK cells [7]. However, abscopal effect allows the immune system restoration. In this light, it can be hypothesized that standard radiotherapy doses, which usually classified as high-dose, may cause malignant cells to expose or release their sequestered antigens that had been previously masked. These tumor antigens can be recognized by cytotoxic $\mathrm{T}$ cells which in turn circulate through the blood stream and destroy remaining tumor cells in different parts of the body. Accordingly, abscopal effect is reported to be blocked by immunosuppressive drugs or $\mathrm{T}$ cell depletion in animal models [8]. Moreover, Liu et al. reported decreased percentage and absolute count of Tregs following low-dose total body irradiation which successively remove the suppressive effect of these cells on other cancer specific effector cells and let the immune system to resume its anti-cancer activity [9].

\section{Material and Methods}

Relevant papers are reviewed by our research team. All reviewers had expertise in their field of study and experience of reviewing for highimpact international journals.

\section{Results}

Standard radiotherapy doses, which usually classified as high-dose, may cause cancer cells to expose or release their sequestered antigens that had been previously masked. Low dose total-body irradiation (TBI) alone (without standard localized high dose radiotherapy) may cause suppression of distant metastasis of tumor cells. Induction of a "whole body abscopal effect" can be involved in suppression of distant metastasis. The suppressive effect of regulatory $\mathrm{T}$ cells involved in down modulating the immune responses against cancers can also be triggered by radiotherapy.

Immunological of and

\section{its}

\section{Carcinogenesis shortcomings}

Palmer et al. in their recently published article titled "thymic involution and rising disease incidence with age" [10] discussed about the accumulation of somatic mutations versus age-related insufficiency of immune system in the induction/progression of malignancies. This model blames the decline of the immune system as the key link between age and cancer. The major shortcomings of the latter model has been discussed by Mortazavi [11]. He believes that, regardless of the fact that the incidence of cancers predominantly increases with age, cancer rate may decline or reach to plateau in very elderly. Furthermore, some malig- 
Abscopal effect following cancer radiation therapy

nancies such as leukemia are more common among children, when the immune system is expected to work well. Considering the link between abscopal effect and mechanisms involving the immune responses, Ngwa et al. have recently raised the importance of combining radiotherapy with immunotherapy to provide an opportunity to boost the abscopal effect [2].

\section{The Issue of Chemotherapy}

Early on, many patients who had their primary cancers treated with radiation were observed to develop metastasis at other locations. Although some scientists believe that with the reduction of primary tumor size following radiotherapy, metastatic spreading would be reduced, there are some evidence regarding radiation-induced metastasis via direct release of cancer cells into circulation or changes in cancer cell phenotype which might be more resistant to different therapeutic strategies [12]. Furthermore, radiotherapy-induced inflammation has been considered as a possible factor in tumor progression [13,14].

In contrast to undesirable cancer-specific immune response following combination of radiotherapy and chemotherapy, concurrent or successive combination of radiotherapy and immunotherapy specially using checkpoint inhibitors is more promising $[15,16]$.

Because of microscopic size of some metastatic foci at the time of radiotherapy, they might be missed and not be targeted for direct radiotherapy. This is the reason it became standard practice to use a combination of chemotherapy and radiation therapy in order to more efficient elimination of unknown metastatic malignant cells.

In addition to temporary effect of chemotherapy, drug-resistant remaining malignant cells may induce a more progressive cancer. Moreover, antimetabolic chemotherapeutic drugs not only kill dividing cancer cells, but also destroy proliferating cancer-specific immune cells. It is worth considering in the case report by Azami et al. that their patient "did not receive chemotherapy due to her poor performance status" and lack of chemotherapy made the cancer treatment successful in this patient. It might be assumed that as one of the side effects of chemotherapy is the suppression of immune responses.

It can be expected that, at least in some cases, cancer treatment would be more efficient in the absence of chemotherapy. However, thousands of patients annually decide not to undergo any kind of chemotherapy or other treatments, but very few miracles occur! On the other hand, in the presence of immune system boosters like adjuvants a greater cancer-specific immuneresponse can be produced $[17,18]$.

\section{Why Are These Miracles So Rare?}

Welsh and his colleagues have reported that patients with melanoma, on rare occasions, can spontaneously go into remission [19,20]. However, partial spontaneous regressions of melanoma lesions are reported to be more common. Although scientists are not fully aware of the causes of these effects, some evidence indicates the role of immune system in attacking cancer cells. Welsh believes that the role of the immune system in regressions of melanoma is due to the induction of abscopal effect. Given this consideration, a localized treatment such as radiation therapy may have two effects; shrinking the targeted tumor as well as stimulating the immune system to mount a systemic attack on cancer cells [20].

If we postulate that in the two cases reported by Azami [5] and Welsh [6], the abscopal effect was the only mechanism behind the eradication of cancer and assume that abscopal effect is only caused by the 
activation of immune system, a key question would be why these cases are only limited to a few patients. Radiation therapy is immune suppressive because it gives high radiation dose to the target tumor mass and its near area, meanwhile other parts of the body receive lower doses of radiation that may stimulate the immune system. As it seems, immune suppressive effect overwhelms the immune boosting effect most of the time and so abscopal effect is rarely observed.

\section{A Challenging Alternative: Ab- scopal Effect Resolves the Prob- lem of Immune Suppression}

Although it seems to be very challenging, Sakamoto reported that combination of the localized high-dose radiotherapy and low doses of total-body irradiation (TBI) enhanced tumor cell killing compared with only local irradiation [21]. He showed that although tumor-cell killing effect was not observed following 10 or 15 cGy of TBI alone, the suppression of distant metastasis of tumor cells was observed following low doses of TBI alone. It can be hypothesized that, in this experiment TBI caused a "whole body abscopal effect". Considering current limited data, it is too early to draw any conclusion about the clinical outcome of this challenging approach as an alternative treatment.

\section{Discussion}

The abscopal effect and its potential importance. Although there is currently no definitive explanation for this effect, there are possible explanations that arise from studies of the effects of low dose radiation. As noted by Welsh [6], radiotherapy deposits high dose radiation in the vicinity of the target tissue, but also leads to lower radiation doses in areas outside the target tissue. This low dose exposure has the effect of stimulating the human immune system and triggers an integrated systemic response to the bodily stress. This stress, caused by radiation or chemotherapy, triggers an integrated response that includes a collection of repair and defense mechanisms including: adaptive response, immune system repair and mitigation, apoptosis, and other inherent repair mechanisms have a result that can be collectively described as an abscopal effect. Although simplistic, this integrated description provides a first order explanation of the abscopal effect and may lead to a more comprehensive explanation and route for future studies to further understand this important effect.

\section{Conclusion}

The occurrence of abscopal effect after radiotherapy can be a key factor for inducing anticancer response in metastatic malignant lesions. Not receiving chemotherapy is also reported to increase the chance of cancer regression after radiotherapy. Given this consideration, standard radiotherapy doses (usually classified as high-dose) may cause cancer cells to expose or release their sequestered antigens that had been previously masked. The suppressive effect of regulatory $\mathrm{T}$ cells involved in down modulating the immune responses against cancers can also be triggered by radiotherapy. In summary, it can be concluded that cancer treatments would be more successful if immune system is boosted not destroyed by the treatments.

\section{Footnotes}

SMJ Mortazavi and Shirin Farjadian equally contributed to this work.

\section{Conflict of Interest}

None 


\section{References}

1. Golden EB, Demaria S, Schiff PB, Chachoua A, Formenti SC. An abscopal response to radiation and ipilimumab in a patient with metastatic non-small cell lung cancer. Cancer Immunol Res. 2013;1:365-72. doi: 10.1158/2326-6066.CIR-13-0115. PubMed PMID:24563870.PubMedPMCID:PMC3930458.

2. Ngwa W, Irabor OC, Schoenfeld JD, Hesser J, Demaria S, Formenti SC. Using immunotherapy to boost the abscopal effect. Nat Rev Cancer. 2018;18:313-22. doi: 10.1038/nrc.2018.6. PubMed PMID: 29449659. PubMed PMCID: PMC5912991.

3. Pleyers C, Piret P, Rorive A, Coucke PA. Could we consider that radiotherapy is effective outside the irradiation area? The abscopal effec]. Rev Med Liege. 2018;73:17-21. PubMed PMID: 29388406.

4. Pouget JP, Georgakilas AG, Ravanat JL. Targeted and Off-Target (Bystander and Abscopal) Effects of Radiation Therapy: Redox Mechanisms and Risk/Benefit Analysis. Antioxid Redox Signal. 2018;29:1447-87. doi: 10.1089/ars.2017.7267. PubMed PMID: 29350049. PubMed PMCID: PMC6199630.

5. Azami A, Suzuki N, Azami Y, Seto I, Sato A, Takano Y, et al. Abscopal effect following radiation monotherapy in breast cancer: A case report. Mol Clin Oncol. 2018;9:283-6. doi: 10.3892/mco.2018.1677. PubMed PMID: 30155251. PubMed PMCID: PMC6109680.

6. Welsh J. Disappearing act: an athletic young man's skin cancer is spreading rapidly and is likely to be fatal-then his health takes a strange turn. Discover Magazine. 2014:24-6.

7. Li F, Zhao Y, Wei L, Li S, Liu J. Tumor-infiltrating Treg, MDSC, and IDO expression associated with outcomes of neoadjuvant chemotherapy of breast cancer. Cancer Biol Ther. 2018;19:695705. doi: 10.1080/15384047.2018.1450116. PubMed PMID: 29621426. PubMed PMCID: PMC6067871.

8. Zhang X, Niedermann G. Abscopal Effects With Hypofractionated Schedules Extending Into the Effector Phase of the TumorSpecific T-Cell Response. Int J Radiat Oncol Biol Phys. 2018;101:63-73. doi: 10.1016/j. ijrobp.2018.01.094. PubMed PMID: 29534901.
9. Liu R, Xiong S, Zhang L, Chu Y. Enhancement of antitumor immunity by low-dose total body irradiationis associated with selectively decreasing the proportion and number of T regulatory cells. Cell Mol Immunol. 2010;7:157-62. doi: 10.1038/cmi.2009.117. PubMed PMID: 20140010. PubMed PMCID: PMC4001896.

10.Palmer S, Albergante L, Blackburn CC, Newman TJ. Thymic involution and rising disease incidence with age. Proc Natl Acad Sci U S A. 2018;115:1883-8. doi: 10.1073/pnas.1714478115. PubMed PMID: 29432166. PubMed PMCID: PMC5828591.

11. Mortazavi SMJ. Shortcomings of the immunological model of carcinogenesis. Proc Natl Acad Sci U S A. 2018;115:E4318. doi: 10.1073/pnas.1802507115. PubMed PMID: 29669916. PubMed PMCID: PMC5949006.

12. Blyth BJ, Cole AJ, MacManus MP, Martin OA. Radiation therapy-induced metastasis: radiobiology and clinical implications. Clin Exp Metastasis. 2018;35:223-36. doi: 10.1007/s10585017-9867-5. PubMed PMID: 29159430.

13.Coskun T, Kosova F, Ari Z, Sakarya A, Kaya Y. Effect of oncological treatment on serum adipocytokine levels in patients with stage II-III breast cancer. Mol Clin Oncol. 2016;4:893-7. doi: 10.3892/mco.2016.815. PubMed PMID: 27123303. PubMed PMCID: PMC4840827.

14.Coussens LM, Werb Z. Inflammation and cancer. Nature. 2002;420:860-7. doi: 10.1038/nature01322. PubMed PMID: 12490959. PubMed PMCID: PMC2803035.

15.Reynders K, Illidge T, Siva S, Chang JY, De Ruysscher D. The abscopal effect of local radiotherapy: using immunotherapy to make a rare event clinically relevant. Cancer Treat Rev. 2015;41:503-10. doi: 10.1016/j.ctrv.2015.03.011. PubMed PMID: 25872878. PubMed PMCID: PMC4816218.

16.Johnson CB, Jagsi R. The Promise of the Abscopal Effect and the Future of Trials Combining Immunotherapy and Radiation Therapy. Int J Radiat Oncol Biol Phys. 2016;95:1254-6. doi: 10.1016/j. ijrobp.2016.02.067. PubMed PMID: 27354132.

17.Du B, Jiang Q-L, Cleveland J, Liu B-R, Zhang D. Targeting Toll-like receptors against cancer. Journal of Cancer Me- 
Welsh J. et al

tastasis and Treatment. 2016;2:464.

18. Lim YT. Vaccine adjuvant materials for cancer immunotherapy and control of infectious disease. Clin Exp Vaccine Res. 2015;4:54-8. doi: 10.7774/cevr.2015.4.1.54. PubMed PMID: 25648865. PubMed PMCID: PMC4313109.

19.Loyola University Health System [Internet]. Combining radiation with immunotherapy showing promise against melanoma. [Cited 19 May 2016]. Available from: www.sciencedaily. com/releases/2016/05/160519151859.htm

20.Stang K, Silva S, Block AM, Welsh JS. The integration of radiation therapy and immunotherapy in melanoma management. Journal of Radiation Oncology. 2016;5:131-42.

21.Sakamoto K. Radiobiological basis for cancer therapy by total or half-body irradiation. Nonlinearity Biol Toxicol Med. 2004;2:293-316. doi: 10.1080/15401420490900254. PubMed PMID: 19330149. PubMed PMCID: PMC2657505. 